\title{
Mode coupling and photon antibunching in a bimodal cavity containing a dipole-quantum-emitter
}

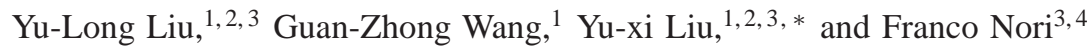 \\ ${ }^{I}$ Institute of Microelectronics, Tsinghua University, Beijing 100084, China \\ ${ }^{2}$ Tsinghua National Laboratory for Information Science and Technology (TNList), Beijing 100084, China \\ ${ }^{3}$ CEMS, RIKEN, Saitama 351-0198, Japan \\ ${ }^{4}$ Department of Physics, The University of Michigan, Ann Arbor, Michigan 48109-1040, USA
}

(Dated: March 6, 2018)

\begin{abstract}
We study the effect of mode-coupling on a single-photon device in which a dipole-quantum-emitter (DQE) is embedded in a bimodal whispering-gallery-mode cavity (WGMC). A scatterer is used to induce mode coupling between counter-clockwise and clockwise propagating light fields, which interact with the DQE. In contrast to models for the interaction between a DQE and a (one-mode or two-mode) cavity field, we find that strong photon antibunching can occur even for a weak DQE-field coupling and large dephasing of the DQE, when mode coupling is introduced. We also find that mode coupling can make the device robust against either the frequency mismatch between cavity modes and the DQE or the coupling strength mismatch between the DQE and each mode in the two-mode cavity. Moreover, we find that these mismatches can be used to generate better antibunching in the weak DQE-field coupling regime. Our study shows that mode coupling in a bimodal cavity is very important for the realization of a good single-photon device.

PACS numbers: 42.50.Ct, 42.50.Ar
\end{abstract}

\section{INTRODUCTION}

Single-photon devices play a crucial role in quantum information science, including quantum communication and quantum computing technology [1, 2]. These devices are useful for quantum key distribution [3, 4], generation of entangled states [5], quantum metrology [6-9], single-photon quantum memory [10-13], linear optical quantum computing [14, 15], and quantum simulations [16, 17]. Thus, a highly-efficient method for generating single-photons with low error is urgently required for quantum information processing. Similar to Coulomb blockade for electrons in mesoscopic electronic devices [18-20], photon blockade, where the subsequent photons are prevented from resonantly entering a cavity, is a promising way to produce single-photon sources. Photon blockade requires that the strength of the photon-photon Kerr nonlinear interaction [21, 22] is much larger than the decay rate of the cavity field.

In principle, the photon blockade can be measured by the second-order correlation functions of photons [23, 24]. Photon blockade in the optical frequency domain has been observed in: a trapped atom coupled to a cavity field [25], a two-level system coupled to a bimodal microtoroidal resonator [26], and a quantum dot coupled to single-mode field of a photonic crystal resonator [27]. Recently, photon blockade in the microwave frequency domain was also observed [28, 29] by coupling a superconducting artificial atom [30-32] to a transmission line resonator. A crucial prerequisite for photon blockade in these experiments [25-29] is to reach the strong field-atom coupling regime [33], i.e., the coupling strength between the cavity field and the dipole-quantum-emitter (DQE) exceeding the decay rates of both the cavity field and DQE. The strong DQE-field coupling can induce a strong nonlinear

\footnotetext{
*Electronic address: yuxiliu@ mail.tsinghua.edu.cn
}

photon-photon interaction and could be used to produce photon blockade and single-photon sources [34-38]. However, when a cavity field works at the single-photon level, it is not easy to achieve a cavity-atom-induced strong photon-photon interaction.

Recently, photonic "molecule" systems, consisting of two coupled cavities with Kerr nonlinearities, were proposed to generate antibunching photon and photon blockade [39]. In contrast to previous studies [25-29], it was found that the photon blockade can occur even in a weak field-atom coupling regime, when an additional cavity is coupled [40]. This new mechanism corresponds to a destructive quantum interference effect in nonlinear photonic molecule systems [40]. Motivated by several studies [39, 40], various systems were proposed to achieve photon blockade and antibunching photons, such as coupled optomechanical systems [41-43], dipolariton systems formed by cavity fields and excitons [44], coherent-feedback controlled optomechanical systems [45], and coupled microcavities with second- or third-order nonlinearities [46, 47]. However, the role of the coupled cavity was not explored in detail. Thus it is interesting to further explore this issue as a function of the coupling strength between the two cavities.

Motivated by an experiment [26] on a photon turnstile, in which a DQE is embeded in a bimodal whispering-gallerymode cavity (WGMC) [26], we here mainly study the mode coupling effect on the nonclassical properties of photons. For two strongly-coupled resonators [39-47], two modes of two resonators are coupled to different nonlinear quantum systems or only one of the cavity modes is coupled to a nonlinear quantum system. However, here: (i) a single WGMC is used to support two degenerate counter-propagating cavity modes; (ii) the mode coupling or normal-mode splitting between these two counter-propagating modes is realized by a scattering object [48-56]; (iii) two cavity modes are coupled to the same DQE. Compared to the studies where a two-level system is coupled to two modes of a cavity [57, 58], here the mode coupling is introduced by a scattering object. 


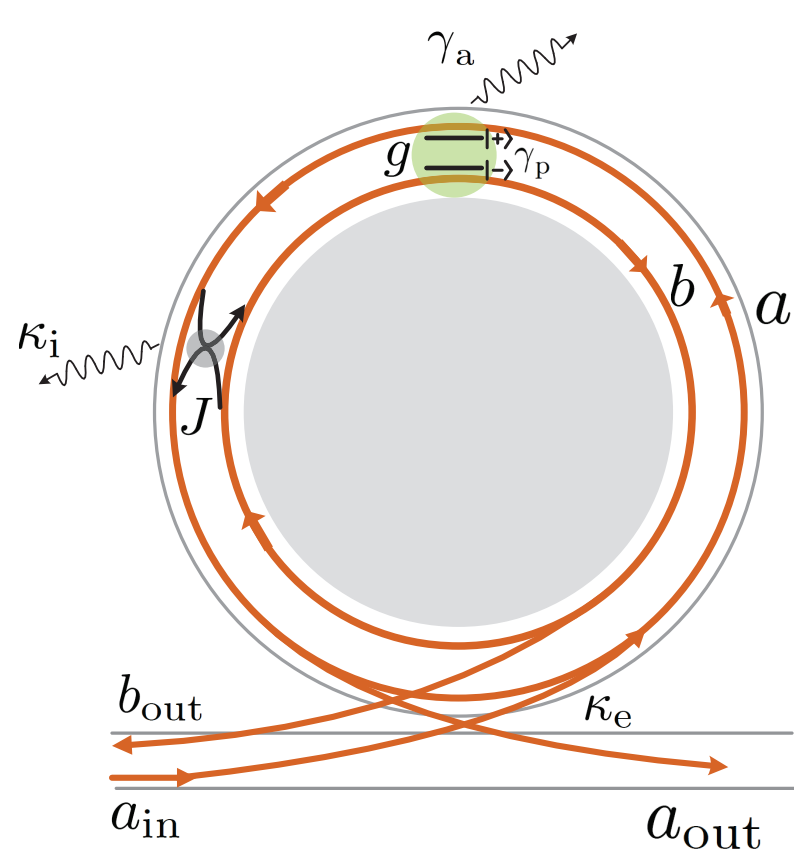

FIG. 1: (Color online) Schematic of a WGMC-DQE system which is driven via an optical fiber taper waveguide with a cavity-waveguide coupling rate $\kappa_{\mathrm{e}}$. The nanoparticle in the WGMC, causes an effective coupling at a rate $J$, between the clockwise and counterclockwise propagating modes. Such two WGM modes have a DQE-field coupling rate $g$ and intrinisc cavity decay rate $\kappa_{\mathrm{i}}$. The DQE has a spontaneous emission rate $\gamma_{\mathrm{a}}$ and a phase dephasing rate $\gamma_{\mathrm{p}}$.

The remaining part of the paper is organized as follows. In Sec. II, the theoretical model is introduced. In Sec. III, the measurements for the mode-coupling are discussed. In Sec. IV, the photon blockade is analytically and numerically studied in different parameter regimes via the second-order correlation function. In particular, we analyze the photon blockade in the weak DQE-field coupling regime. We also compare our results with those in coupled-cavity systems [3947]. In particular, we analyze the physical mechanism of the photon blockade in the system studied here. In Sec. V, we study the robustness of the system on the dephasing of the DQE. In Sec. VI, we study the robustness of the system on either the frequency mismatches between the cavity mode and the DQE or the coupling strength mismatch between the DQE and each cavity mode. Conclusions and perspective discussions are finally presented in Sec. VII.

\section{HAMILTONIAN}

As schematically shown in Fig. 11, we study a system that consists of a single whispering-gallery-mode cavity (WGMC) and a dipole-quantum-emitter (DQE). As studied in Ref. [26], the WGMC supports a counterclockwise (CCW) and a clockwise $(\mathrm{CW})$ propagating modes which have the same frequency. These two modes are coupled to each other through a nanoparticle (called as a scatterer) The DQE is modeled as a two-level system, which can be a quantum-dot, an atom, or other systems. Here we do not focus on any particular system, but consider a generic DQE. The Hamiltonian of the whole system is given by [26, 55, 56]

$$
\begin{aligned}
H & =\hbar \omega\left(a^{\dagger} a+b^{\dagger} b\right)+\hbar \omega_{\mathrm{a}} \sigma_{z}+\hbar J\left(a^{\dagger} b+b^{\dagger} a\right) \\
& +\hbar\left[g_{a} a^{\dagger} \sigma_{-}+g_{b} b^{\dagger} \sigma_{-}+\varepsilon a^{\dagger} e^{-i \omega_{\mathrm{d}} t}+\text { H.c. }\right],
\end{aligned}
$$

where $a$ and $b\left(a^{\dagger}\right.$ and $\left.b^{\dagger}\right)$ are the annihilation (creation) operators of $\mathrm{CCW}$ and $\mathrm{CW}$ propagating modes with frequency $\omega$, respectively. $\sigma_{-}$and its conjugate operator $\sigma_{+}$are the ladder operators describing a DQE with frequency $\omega_{a}$, and $J$ is the mode-coupling strength between the $\mathrm{CCW}$ and $\mathrm{CW}$ modes. We assume that the DQE and two propagating modes have coupling strengths $g_{a}$ and $g_{b}$. The driving field is assumed to be coupled to the $\mathrm{CCW}$ mode with the coupling strength $\varepsilon$.

The mode coupling in the WGMC is usually introduced when a scatterer is present. The scatterer can be considered as a nanoparticle, which is used to enhance [50] and control [51] the effective coupling strength $J$ between the $\mathrm{CCW}$ and $\mathrm{CW}$ modes. Let us consider a simple example where the nanoparticle is assumed to be a nanosphere with radius $R \ll \lambda$, where $\lambda$ is the wavelength of the light field. Then the response of a particle to an electromagnetic field can be calculated by the Clausius-Mossotti relation and the effective coupling strength $J$ becomes [54]

$$
J=-\frac{1}{2 V} \alpha f^{2} \omega,
$$

in the electrostatic limit, with

$$
\alpha=4 \pi R^{3}\left(\frac{n_{\mathrm{p}}^{2}-1}{n_{\mathrm{p}}^{2}+2}\right) .
$$

Here $f$ is the mode function of the WGMC, $V$ is the mode volume of the WGMC, $n_{p}$ is the refractive index of the particle, and the surrounding medium is assumed to be air.

The scatterer-induced mode splitting has been observed experimentally, and proposed for a highly sensitive and robust platform for detecting nanoscale objects [48-54]. As reported in [48], a nanoparticle of radius $40 \mathrm{~nm}$ can introduce an apparent mode splitting with a mode-coupling strength $J>\kappa$, where $\kappa$ is the decay rate of the cavity field, producing a strong-mode coupling. From Eqs. (2) and (3), it is clear that the mode-coupling strength $J$ can be enhanced by increasing the radius of the nanoparticle. The effective coupling strength can also be enhanced by increasing the number of nanoparticles [54], improving the quality factor of the microresonator [50], introducing gain medium into the cavity [53], or using Raman-gain-induced loss compensation [62]. Here, we will not pay more attention to how the nanoparticle will affect the system. Instead, we just assume that the mode-coupling strength $J$ is a positive and real number, which can be controlled and set to be much larger than the decay rate $\kappa$ of the cavity field. We will mainly focus on the study of how the mode-coupling strength $J$ affects the single-photon behavior in the system.

In the rotating reference frame at the driving-field frequency $\omega_{\text {d }}$, i.e., with a unitary transformation $U=$ 


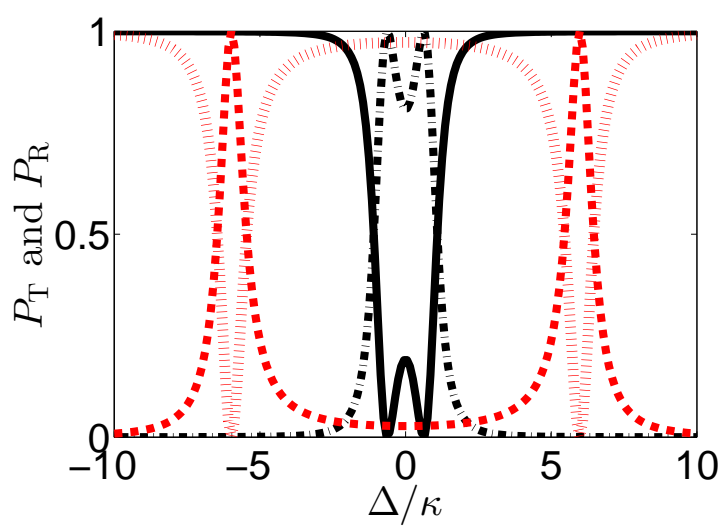

FIG. 2: (Color online) Normalized transmission spectra $P_{\mathrm{T}}$ and reflection spectra $P_{\mathrm{R}}$ of the coupled WGMC-mode system. The horizontal axis shows the normalized $\Delta=\omega-\omega_{\mathrm{d}}$, which is the detuning from the driving frequency $\omega_{\mathrm{d}}$. Also, $\kappa$ is the decay rate of the cavity field. Two different parameter regimes are shown: (i) when the optical mode-coupling rate $J$ is much larger than the cavity-field linewidth $\kappa$, corresponding to the red-dotted curve for $P_{\mathrm{T}}$ and reddashed curve for $P_{\mathrm{R}}$, with $J=6 \kappa$ as an example; and also (ii) when $J$ is comparable to $\kappa$, corresponding to the black-solid curve for $P_{\mathrm{T}}$ and black-dash-dotted curve for $P_{\mathrm{R}}$, with $J=0.8 \kappa$ as an example.

$\exp \left[-i \omega_{\mathrm{d}}\left(a^{\dagger} a+b^{\dagger} b+\sigma_{z}\right) t\right]$, the Hamiltonian in Eq. (1) is given as

$$
\begin{aligned}
H_{\mathrm{eff}} & =\hbar \Delta\left(a^{\dagger} a+b^{\dagger} b\right)+\hbar \Delta_{\mathrm{a}} \sigma_{z}+\hbar J\left(a^{\dagger} b+b^{\dagger} a\right) \\
& +\hbar\left[g\left(a^{\dagger}+b^{\dagger}\right) \sigma_{-}+\varepsilon a^{\dagger}+\text { H.c. }\right],
\end{aligned}
$$

with the detunings $\Delta=\omega-\omega_{\mathrm{d}}$ and $\Delta_{\mathrm{a}}=\omega_{\mathrm{a}}-\omega_{\mathrm{d}}$, from the driving field $\omega_{\mathrm{d}}$ to the cavity modes $\omega$ and to the DQE $\omega_{\mathrm{a}}$. Below, we first discuss the case for $g_{\mathrm{a}} \equiv g_{\mathrm{b}} \equiv g$ and $\Delta_{\mathrm{a}}=\Delta$ (i.e., $\omega=\omega_{\mathrm{a}}$ ) in Secs. III, IV, and V. The mismatch of the coupling strengths $g_{\mathrm{a}}$ and $g_{\mathrm{b}}$ and the mismatch of the frequencies $\omega$ and $\omega_{\mathrm{a}}$ will be discussed in Sec. VI.

\section{MEASUREMENT OF THE MODE-COUPLING STRENGTH}

Let us first study how the mode-coupling strength $J$ could be measured. The dynamics of the whole system is described by the quantum Langevin equations

$$
\begin{aligned}
\frac{d a}{d t} & =-\left(i \Delta+\frac{\kappa}{2}\right) a-i J b-i g \sigma_{-}-i \varepsilon-\sqrt{\kappa} a_{\mathrm{in}}, \\
\frac{d b}{d t} & =-\left(i \Delta+\frac{\kappa}{2}\right) b-i J a-i g \sigma_{-}-\sqrt{\kappa} b_{\mathrm{in}}, \\
\frac{d \sigma_{-}}{d t} & =-\left(i \Delta+\frac{\gamma}{2}\right) \sigma_{-}+i g a \sigma_{\mathrm{z}}+i g b \sigma_{\mathrm{z}}-\sqrt{\gamma} \sigma_{\mathrm{in}} .
\end{aligned}
$$

Here, the decay rates of the two cavity modes are denoted by $\kappa$, which consists of the intrinsic loss $\kappa_{\mathrm{i}}$ and the external loss $\kappa_{\mathrm{e}}$, i.e., $\kappa=\kappa_{\mathrm{i}}+\kappa_{\mathrm{e}}$. For the DQE, the decay rate $\gamma$ consists of the spontaneous emission rate $\gamma_{a}$ and phase dephasing rate $\gamma_{\mathrm{p}}$, i.e., $\gamma=\gamma_{\mathrm{a}}+2 \gamma_{\mathrm{p}}$. Here, $a_{\mathrm{in}}, b_{\mathrm{in}}$ and $\sigma_{\text {in }}$ are the noise operators associated with the $\mathrm{CCW}$ mode, $\mathrm{CW}$ mode, and the $\mathrm{DQE}$ with zero mean-value, i.e., $\left\langle a_{\text {in }}\right\rangle=\left\langle b_{\text {in }}\right\rangle=\left\langle\sigma_{\text {in }}\right\rangle=0$.

The mode coupling can be clearly observed in the transmitted $\left(P_{\mathrm{T}}\right)$ and reflected $\left(P_{\mathrm{R}}\right)$ cavity field power. Let us now calculate these through the outputs of the modes $a$ and $b$, respectively. Using the mean field approximation and from Eqs. (5) and (6), the equations of motion for the mean value of each operator can be given as

$$
\begin{aligned}
\frac{d}{d t}\langle a\rangle & =-\left(i \Delta+\frac{\kappa}{2}\right)\langle a\rangle-i J\langle b\rangle-i g\left\langle\sigma_{-}\right\rangle-i \varepsilon, \\
\frac{d}{d t}\langle b\rangle & =-\left(i \Delta+\frac{\kappa}{2}\right)\langle b\rangle-i J\langle a\rangle-i g\left\langle\sigma_{-}\right\rangle, \\
\frac{d}{d t}\left\langle\sigma_{-}\right\rangle & =-\left(i \Delta+\frac{\gamma}{2}\right)\langle\sigma\rangle+i g(\langle a\rangle+\langle b\rangle)\left\langle\sigma_{z}\right\rangle .
\end{aligned}
$$

Using the input-output theory [63, 64], the output of each mode can be obtained as

$$
\begin{aligned}
& \left\langle a_{\text {out }}\right\rangle=\frac{i \varepsilon}{\sqrt{\kappa}}+\sqrt{\kappa}\langle a\rangle, \\
& \left\langle b_{\text {out }}\right\rangle=\sqrt{\kappa}\langle b\rangle .
\end{aligned}
$$

Here, we are only interested in the mode coupling induced by the scatterer, thus we can set $g=0$. Then the dynamical equations of the cavity modes are simplified to

$$
\begin{aligned}
& \frac{d}{d t}\langle a\rangle=-\left(i \Delta+\frac{\kappa}{2}\right)\langle a\rangle-i J\langle b\rangle-i \varepsilon, \\
& \frac{d}{d t}\langle b\rangle=-\left(i \Delta+\frac{\kappa}{2}\right)\langle b\rangle-i J\langle a\rangle .
\end{aligned}
$$

By solving Eqs. (13) and (14) in the steady-state with $\langle\dot{a}\rangle=$ $\langle\dot{b}\rangle=0$, we have

$$
\begin{aligned}
& \langle a\rangle=\frac{\Delta-i \frac{\kappa}{2}}{\left(i \Delta+\frac{\kappa}{2}\right)^{2}+J^{2}} \varepsilon, \\
& \langle b\rangle=\frac{-J}{\left(i \Delta+\frac{\kappa}{2}\right)^{2}+J^{2}} \varepsilon .
\end{aligned}
$$

Combining Eqs. (11) and (12) with the steady-state solutions, the normalized transmission power $P_{\mathrm{T}}$ and reflection power $P_{\mathrm{R}}$ from the WGMC are given by

$$
\begin{aligned}
& P_{\mathrm{T}}=\frac{1}{\varepsilon^{2}}\left|\left\langle a_{\text {out }}\right\rangle\right|^{2}=\left|\frac{i}{\sqrt{\kappa}}+\frac{\sqrt{\kappa}\left(\Delta-i \frac{\kappa}{2}\right)}{\left(i \Delta+\frac{\kappa}{2}\right)^{2}+J^{2}}\right|^{2}, \\
& P_{\mathrm{R}}=\frac{1}{\varepsilon^{2}}\left|\left\langle b_{\text {out }}\right\rangle\right|^{2}=\left|\frac{\sqrt{\kappa} J}{\left(i \Delta+\frac{\kappa}{2}\right)^{2}+J^{2}}\right|^{2} .
\end{aligned}
$$

In Fig. 2, the transmission $P_{\mathrm{T}}$ and reflection $P_{\mathrm{R}}$ powers are plotted as a function of the detuning parameter $\Delta=\omega-\omega_{\mathrm{d}}$. Two different parameter regimes are discussed: (i) $J>\kappa$ and (ii) $J \sim \kappa$. For $J>\kappa$, it is obvious that a pair of resonances appear and are located at $\Delta \pm J$. Actually, these resonances correspond to two different standing light waves, which are superpositions (i.e. $(a+b) / \sqrt{2}$ and $(a-b) / \sqrt{2})$ 
of the two counter-propagating cavity modes. When the coupling strength $J$ is much larger than $\kappa$, a well-resolved modesplitting can be clearly observed in the transmission or reflection spectra. However, the distance between two resonances becomes close when $J \sim \kappa$, and is no longer distinguishable when $J \ll \kappa$.

\section{PHOTON BLOCKADE IN THE WEAK DQE-FIELD COUPLING REGIME}

In this section we will study the photon blockade. We mainly study how the quantum behavior of the mode fields in the WGMC varies with the change of the mode-coupling strength $J$ between the two modes. Compared with previous studies on the strong coupling between a two-level atom and a single-mode cavity (e.g., in Refs. [25-29]) or a bimodal cavity without the mode coupling [57, 58], we will here focus on the weak coupling between a DQE and the cavity modes with mode coupling.

\section{A. Master equation and second-order correlation functions}

By taking the dissipation into account, we can write the master equation [59-61] of the reduced density matrix $\rho$ for whole system as

$$
\begin{aligned}
\frac{d \rho}{d t} & =\frac{1}{i \hbar}\left[H_{\mathrm{eff}}, \rho\right]+\frac{\kappa}{2}\left(2 a \rho a^{\dagger}-a^{\dagger} a \rho-\rho a^{\dagger} a\right) \\
& +\frac{\kappa}{2}\left(2 b \rho b^{\dagger}-b^{\dagger} b \rho-\rho b^{\dagger} b\right) \\
& +\frac{\gamma_{\mathrm{a}}}{2}\left(2 \sigma \rho \sigma^{\dagger}-\sigma^{\dagger} \sigma \rho-\rho \sigma^{\dagger} \sigma\right) \\
& +\frac{\gamma_{\mathrm{p}}}{2}\left(2 \sigma_{z} \rho \sigma_{z}-\rho\right),
\end{aligned}
$$

in the Markov approximation. Here, $H_{\text {eff }}$ is given in Eq. (4). We assume that the cavity fields and the DQE are in a zerotemperature environment, to simplify the calculation. In the number states basis $\left|n_{\mathrm{a}}, n_{\mathrm{b}}, i\right\rangle$ and $\left|n_{\mathrm{a}^{\prime}}, n_{\mathrm{b}^{\prime}}, i^{\prime}\right\rangle$, the formal solution of $\rho$ in Eq. (19) can be given as [39]:

$$
\rho(t)=\sum_{n_{a}, n_{b}} \sum_{n_{a^{\prime}}, n_{b^{\prime}}} \sum_{i, i^{\prime}} \rho_{n_{a}, n_{b}, i ; n_{a^{\prime}}, n_{b^{\prime}}, i^{\prime}}\left|n_{a}, n_{b}, i\right\rangle\left\langle n_{a^{\prime}}, n_{b^{\prime}} i^{\prime}\right| .
$$

where $n_{a}\left(n_{a^{\prime}}\right)$ represents the photon number of the CCW mode, $n_{b}\left(n_{b^{\prime}}\right)$ represents the photon number of the $\mathrm{CW}$ mode and $i\left(i^{\prime}\right)=+,-$ represents the excited and ground state of the DQE, respectively.

We can obtain the steady-state solution $\rho_{s s}$ of the reduced density operator $\rho$ by setting $d \rho / d t=0$, and then the statistical properties of the driven $\mathrm{CCW}$ propagating cavity mode in the WGMC can be studied via the normalized equal-time second-order correlation function

$$
g_{\mathrm{CCW}}^{(2)}(0)=\frac{\left\langle a^{\dagger} a^{\dagger} a a\right\rangle}{\left\langle a^{\dagger} a\right\rangle^{2}}=\frac{\operatorname{Tr}\left(\rho_{\mathrm{ss}} a^{\dagger} a^{\dagger} a a\right)}{\left[\operatorname{Tr}\left(\rho_{\mathrm{ss}} a^{\dagger} a\right)\right]^{2}} .
$$

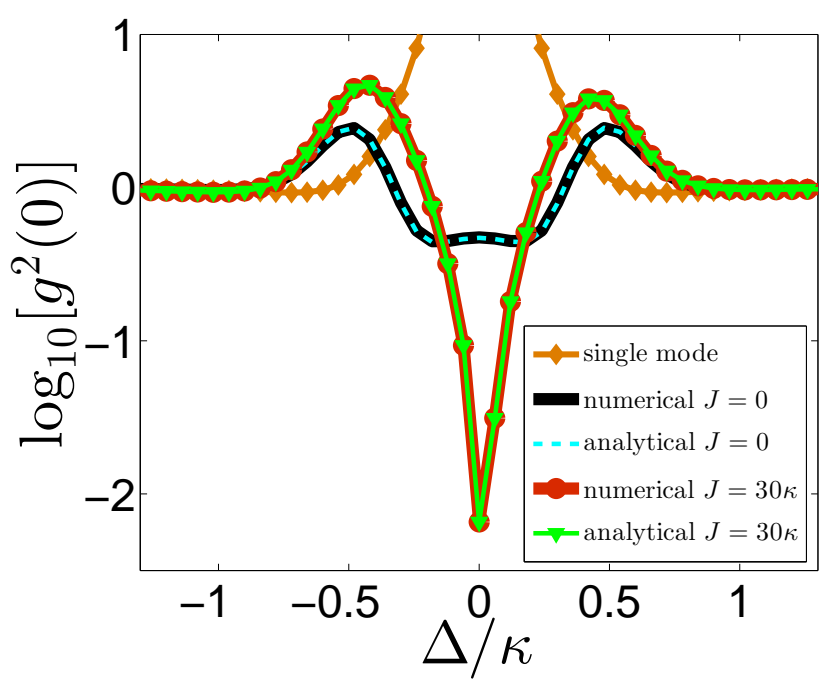

FIG. 3: (Color online) The second-order correlation functions $g_{\mathrm{a}}^{(2)}(0)$ of a CCW propagating intercavity field versus the detuning parameter $\Delta / \kappa=\left(\omega-\omega_{\mathrm{d}}\right) / \kappa$, for a number of different parameters. These correlations are calculated numerically using the master equation and also analytically using the Schröedinger equation in the steady-state. The $g_{\mathrm{a}}^{(2)}(0)$ is shown as an orange-rhombus solid curve for the interaction between a DQE and a single-mode cavity field; The $g_{\mathrm{a}}^{(2)}(0)$ is shown as a black solid curve (numerically calculated) and a cyan-dotted line (analytically calculated) for the interaction between a DQE and two-mode cavity fields with $J=0$. The $g_{\mathrm{a}}^{(2)}(0)$ is shown as a red-circular solid curve (numerically calculated) and a green-triangle solid curve (analytically calculated) for the interaction between a DQE and two-mode cavity fields with $J=30 \kappa$. The system parameters for this simulation are: $\kappa=40 \gamma_{\mathrm{a}}, g=20 \gamma_{\mathrm{a}}$, $\varepsilon=\gamma_{\mathrm{a}}$, and $\gamma_{\mathrm{a}}=1 \mathrm{MHz}$.

In this section, we first study the antibunching effect of the CCW mode by setting $\gamma_{p}=0$, in order to consider the same environmental effect as in previous studies [26]. The effect of dephasing will be studied in Sec. V. We point out that the statistical properties of the $\mathrm{CW}$ mode can also be studied in a similar way as those for the CCW mode.

\section{B. Numerical calculations}

We first study how the second-order correlation functions $g_{\mathrm{CCW}}^{(2)}(0)$ vary with the detuning $\Delta=\omega-\omega_{\mathrm{d}}$ between the driving field and the cavity modes, and also show how the statistical properties of the cavity field are changed by the modecoupling strength $J$. In Fig. 3, $g_{\mathrm{CCW}}^{(2)}(0)$ versus the detuning $\Delta$ is plotted in the DQE-field weak-coupling regime for a DQE interacting with either: (i) a single-mode cavity field, or (ii) bimodal cavity fields without mode coupling, or (iii) bimodal cavity fields with mode coupling. Figure 3 clearly shows that there is only photon bunching when only a singlemode cavity field is coupled to the DQE. This is due to the weak DQE-field coupling, i.e., $g<\kappa$. When the two modes 

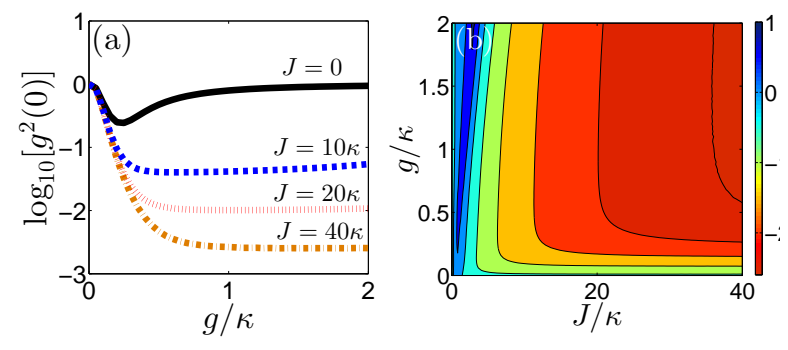

FIG. 4: (Color online) (a) The logarithmic of second-order correlation functions $g_{\mathrm{a}}^{(2)}(0)$ about the CCW-propagating intercavity field, versus DQE-field coupling strength parameter $g / \kappa$, are calculated and shown with different mode-coupling strengths: black-solid line for $J=0$; blue-dashed line for $J=10 \kappa$; red-dotted line for $J=20 \kappa$, and yellow-dot-dashed line for $J=40 \kappa$. (b) The secondorder correlation function $g_{\mathrm{a}}^{(2)}(0)$ of the CCW-propagating intercavity field is plotted as functions of both the mode-coupling strengths $J / \kappa$ and the DQE-field coupling strength parameter $g / \kappa$. The other system parameters for this simulation are: $\kappa=40 \gamma_{\mathrm{a}}, \Delta=0$, $\varepsilon=\gamma_{\mathrm{a}}$, and $\gamma_{\mathrm{a}}=1 \mathrm{MHz}$.

without the mode coupling in the WGMC are coupled to the $\mathrm{DQE}$, the photon antibunching is observed around $\Delta=0$. Such antibunching in the weak DQE-field coupling regime results from an interference between the coherent light transmitted through the resonant cavities and the super-Poissonian light generated by photon-induced tunneling [57]. Thus a balance between the decay rate $\kappa$ of the cavity modes and the DQE-field coupling $g$ is required. When the DQE-field coupling is not in the strong-coupling regime, the antibunching effect is weak, e.g., the minimum value of $g_{\mathrm{CCW}}^{(2)}(0)$ is about 0.47 at $\Delta=0$ for $\kappa=2 g$, as shown in Fig. 3. However, when the mode coupling $J$ is introduced, an obvious minimum and valley appear around $\Delta=0$, e.g., the minimum value $g_{\mathrm{CCW}}^{(2)}(0)$ reaches 0.006 at $\Delta=0$, in contrast to the case when there is no mode coupling. This strong antibunching is due to the mode coupling, which will be discussed in the following paragraphs.

To clearly show how the DQE-field coupling strength $g$ affects the antibunching for different mode-coupling strengths $J$, including $J=0, g_{\mathrm{CCW}}^{(2)}(0)$ versus $g$ is plotted in Fig. 4( $(\mathrm{a})$ for different values of $J$. As shown in Fig. (4) a), for no modecoupling, i.e., $J=0$, we find: (i) a minimum value of $g_{\mathrm{CCW}}^{(2)}(0)=0.25$ around of $g / \kappa=0.2$, which is in the weak DQE-field coupling regime; (ii) the value of $g_{\mathrm{CCW}}^{(2)}(0)$ increases with increasing $g / \kappa$ further; (iii) the value of $g_{\mathrm{CCW}}^{(2)}(0)$ approaches one when $g / \kappa>0.8$ and the photon antibunching gradually disappears; (iv) the value of $g_{\mathrm{CCW}}^{(2)}(0)$ equals to one and the photon antibunching does not occur when $g / \kappa>1$, which is in the strong DQE-field coupling regime. This is counterintuitive with the fact that the better photon antibunching (i.e., the smaller value of $g_{\mathrm{CCW}}^{(2)}(0)$ ) corresponds to a larger $g$. The physical mechanism for this effect has been explained in Ref. [57]. Therefore, a balance between $g$ and $\kappa$ is required, which might not be easy to achieve experimentally. For ex-

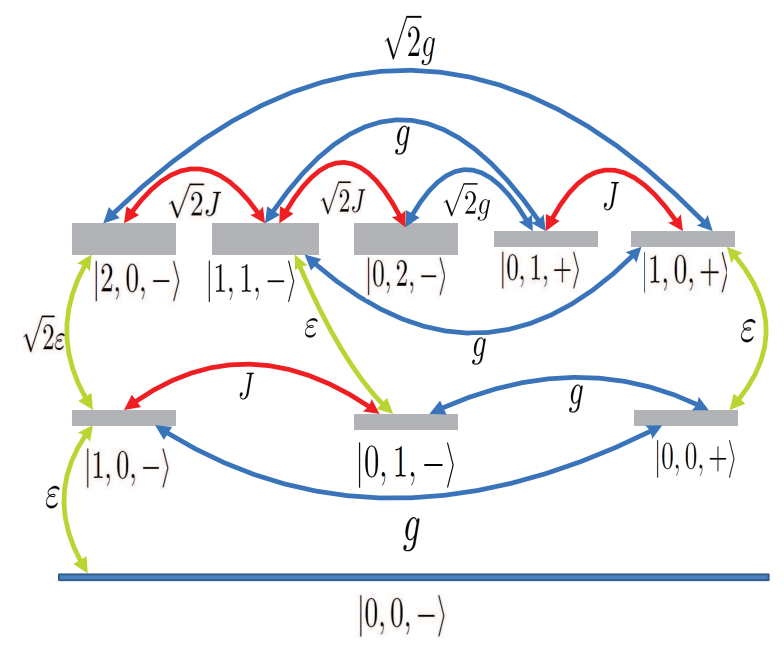

FIG. 5: (Coloronline) Schematic diagram of the energy levels of the WGMC-DQE system corresponding to Fock states up to twophotons. The first photon and second photon indexes in the ket correspond to the photon number in the CCW propagating mode and the $\mathrm{CW}$ propagating mode, respectively. The index $+(-)$ indicate the excited state (ground state) of the DQE. The long blue solid line at the bottom denotes the ground state of the whole system. The narrower short gray lines indicate single-photon states with decay rate $\kappa$, and the wider short gray lines at the top represent two-photon states with decay rate $2 \kappa$. Here we have neglected the decay of the DQE due to the fact that $\kappa \gg \gamma_{a}$. The four green double-arrows represent the driving optical pump for the CCW propagating mode. The six blue double-arrows represent different energy level transitions induced by the DQE-field coupling. The four red duble arrows represent different energy level transitions induced by the CCW-CW mode coupling.

ample, photon antibunching can occur only in a narrow region around the dip, e.g., $g / \kappa=0.2$. However, as shown in Fig. 4(a), when mode-coupling is introduced, a better photon antibunching is achieved both in the weak and strong DQEfield coupling regimes. Figure 4 a) clearly shows that the better photon antibunching (smaller value of $g_{\mathrm{CCW}}^{(2)}(0)$ ) corresponds to the stronger mode-coupling strength in both the weak and strong DQE-field coupling regimes. The variations of $g_{\mathrm{CCW}}^{(2)}(0)$ with $g / \kappa$ are similar for different values of $J$.

We now study how the statistical properties of the cavity fields are affected by the mode-coupling strength $J$ and the DQE-field coupling strength $g$ by plotting $g_{\mathrm{CCW}}^{(2)}(0)$ in Fig. 4(b). We find that a strong photon antibunching can be achieved both in the DQE-field weak-coupling and strongcoupling for the strong mode-coupling, e.g., $J / \kappa>5$. For a given $g$, the larger $J$ corresponds to the smaller value of $g_{\mathrm{CCW}}^{(2)}(0)$, and thus a better photon antibunching is achieved. Similarly, for a nonzero mode-coupling strength $J$, the larger $g$ corresponds to the smaller value of $g_{\mathrm{CCW}}^{(2)}(0)$, and thus a better photon antibunching is achieved. From Fig. 4, we also find that a nonzero mode-coupling strength $J$ makes the balance between $g$ and $\kappa$ unnecessary, to obtain a strong photon antibunching. This will be further discussed below. 


\section{Analytical solutions}

To better understand numerical calculations, we now study an analytical solution and compare it with numerical results. We assume that the driving field is very weak so that the total excitation number of the system is no more than two [39-45]. In this case, we use the ansatz

$$
\begin{aligned}
|\varphi(t)\rangle & =C_{0,0,-}|0,0,-\rangle+C_{1,0,-}|1,0,-\rangle+C_{0,1,-}|0,1,-\rangle \\
& +C_{0,0,+}|0,0,+\rangle+C_{2,0,-}|2,0,-\rangle+C_{0,2,-}|0,2,-\rangle \\
& +C_{1,1,-}|1,1,-\rangle+C_{1,0,+}|1,0,+\rangle+C_{0,1,+}|0,1,+\rangle
\end{aligned}
$$

with $C_{i, j, \pm} \equiv C_{i, j, \pm}(t)$, to calculate the steady state of the system. In the weak-driving limit, we have the relation

$$
\begin{aligned}
C_{0,0,-} & \gg C_{1,0,-}, C_{0,1,-}, C_{0,0,+} \\
& \gg C_{2,0,-}, C_{0,2,-}, C_{1,1,-}, C_{1,0,+}, C_{0,1,+}
\end{aligned}
$$

Using the relation in Eq. (23) and combining with Eq. (21), we have

$$
g_{\mathrm{CCW}}^{(2)}(0)=\frac{2\left|C_{2,0,-}\right|^{2}}{\left|C_{1,0,-}\right|^{4}} .
$$

The coefficients $C_{2,0,-}$ and $C_{1,0,-}$ in the steady state can be obtained via the Schrödinger equation

$$
i \hbar \frac{\partial|\varphi\rangle}{\partial t}=\tilde{H}|\varphi\rangle
$$

and $\partial|\varphi\rangle / \partial t=0$. The effective non-hermitian Hamiltonian $\tilde{H}$ in Eq. 25) is given by

$$
\begin{aligned}
\tilde{H} & =\hbar\left(\Delta-i \frac{\kappa}{2}\right)\left(a^{\dagger} a+b^{\dagger} b\right)+\hbar J\left(a^{\dagger} b+b^{\dagger} a\right) \\
& +\hbar\left(\Delta-i \frac{\gamma_{\mathrm{a}}}{2}\right) \sigma_{z}+\hbar\left[g\left(a^{\dagger}+b^{\dagger}\right) \sigma_{-}+\varepsilon a^{\dagger}+\text { H.c. }\right]
\end{aligned}
$$

in the zero temperature approximation. Here, the dephasing of the DQE has been neglected.

Then, replacing $\tilde{H}$ in Eq. 25) by the expression in Eq. (26), we can obtain a set of linear equations. the detailed derivation can be found in Appendix A. By solving these linear equations, we can obtain the coefficients, e.g. $C_{2,0,-}$ and $C_{1,0,-}$, in Eq. (22), then $g_{\mathrm{CCW}}^{(2)}(0)$ is further calculated as

$$
\left.g_{\mathrm{CCW}}^{(2)}(0)\right)=\frac{2\left|C_{2,0,-}\right|^{2}}{\left|C_{1,0,-}\right|^{4}}=\frac{\left|A_{1}\right|^{2}\left|A_{2}\right|^{2}}{\left|A_{3}\right|^{2}\left|A_{4}\right|^{4}}
$$

with

$$
\begin{aligned}
& A_{1}=\Delta_{\mathrm{p}}\left[\Delta_{\mathrm{p}}^{2} \Delta_{\mathrm{d}}+\left(\Delta_{\mathrm{d}} \Delta_{\mathrm{p}}-2 g^{2}\right)\left(\Delta_{\mathrm{d}}+J\right)\right]+g^{4} \\
& A_{2}=\Delta_{\mathrm{d}}\left(J+\Delta_{\mathrm{p}}\right)-2 g^{2} \\
& A_{3}=\left(J+\Delta_{\mathrm{p}}\right)\left(J+\Delta_{\mathrm{p}}+\Delta_{\mathrm{d}}\right)-2 g^{2} \\
& A_{4}=\Delta_{\mathrm{p}} \Delta_{\mathrm{d}}-g^{2}
\end{aligned}
$$

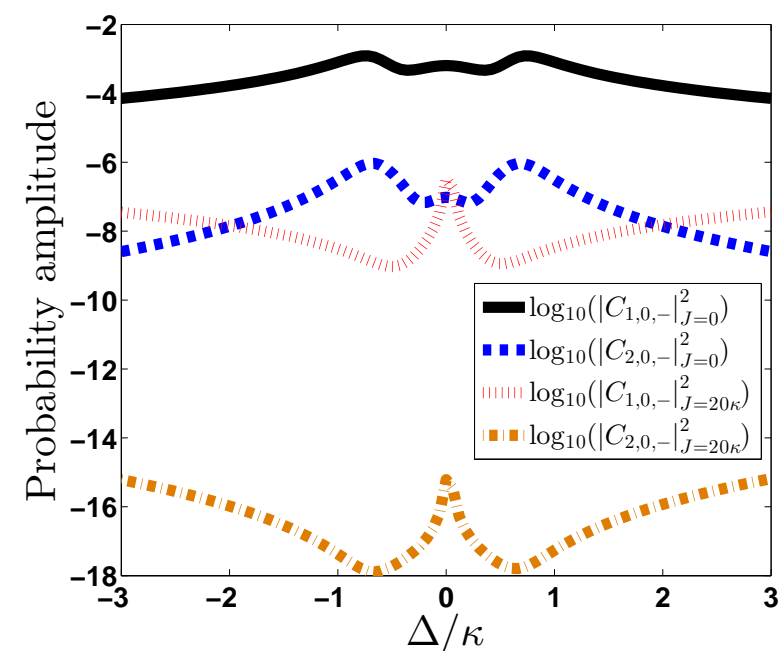

FIG. 6: (Color online) The probability amplitude of the WGMCDQE system with one-photon and two-photons versus the detunning parameter $\Delta / \kappa$ are calculated and shown with different modecoupling strengths: the black-solid curve for the one-photon probability amplitude $\left|C_{1,0,-}\right|^{2}$ and blue-dashed curve for the two-photon probability amplitude $\left|C_{2,0,-}\right|^{2}$ with $J=0$; the red-dotted curve for the one-photon probability amplitude $\left|C_{1,0,-}\right|^{2}$ and the yellow dot dash curve for the two-photon probability amplitude $\left|C_{2,0,-}\right|^{2}$ with $J=20 \kappa$. The other system parameters for this simulation are: $\kappa=40 \gamma_{\mathrm{a}}, g=20 \gamma_{\mathrm{a}}, \varepsilon=\gamma_{\mathrm{a}}$, and $\gamma_{\mathrm{a}}=1 \mathrm{MHz}$.

Here, we define two detunnings by including the dissipation rates

$$
\begin{aligned}
& \Delta_{\mathrm{p}}=\Delta-i \frac{\kappa}{2}, \\
& \Delta_{\mathrm{d}}=\Delta-i \frac{\gamma_{\mathrm{a}}}{2} .
\end{aligned}
$$

For comparison with the numerical calculations using the master equation, $g_{\mathrm{CCW}}^{(2)}(0)$, calculated by analytical solution Eq. (27), versus $\Delta / \kappa$, is also shown in Fig. 3. It is clear that the analytical solutions agree well with the numerical calculations.

\section{Physical mechanism and comparisons between with and without mode coupling}

Using analytical solutions, let us further understand the physical mechanism of the mode-coupling-enhanced photon antibunching. In Fig. 5, we schematically show the energy level structure of the system up to two-particle excitations. In this case, nine states are involved, they are the ground state $|0,0,0\rangle$, three one-particle excitation states $|1,0,-\rangle,|0,1,-\rangle$, and $|0,0,+\rangle$, and five two-particle excitation states $|2,0,-\rangle$, $|0,2,-\rangle,|1,1,-\rangle,|1,0,+\rangle,|0,1,+\rangle$. The connections between different states, due to the driving field $\varepsilon$ and the couplings, characterized by $J$ and $g$, are also schematically shown in Fig. 5. 
We first consider the single-particle excitation. In this case, the CCW mode is pumped to the single-photon state and the system is in the state $|1,0,-\rangle$. When there is no mode coupling, i.e., $J=0$, the probability amplitude $\left|C_{1,0,-}\right|^{2}$, which represents the probability to find the system in the state $|1,0,-\rangle$, is only determined by the decay rate $\kappa$ of the CCW mode and DQE-field coupling strength $g$. In this case and as shown in Fig. 5, there are two transition paths from the state $|1,0,-\rangle$ to the state $|0,0,+\rangle$ or the state $|0,0,-\rangle$. However, when the mode coupling is introduced by the scatter, $\left|C_{1,0,-}\right|^{2}$ depends not only on $\kappa$ and $g$ but also on the mode-coupling strength $J$. Thus an additional transition path between the state $|1,0,-\rangle$ and the state $|0,1,-\rangle$ is introduced by the mode coupling, which may reduce the probability of the system at the state $|1,0,-\rangle$.

When the CCW mode is further pumped to the two-photon state $|2,0,-\rangle$. The additional transition path between the state $|2,0,-\rangle$ and the state $|1,1,-\rangle$ is introduced due to the mode coupling, and this may also reduce the probability of the system in the state $|2,0,-\rangle$. Therefore, when mode coupling $J$ is introduced, both the single-photon probability $\left|C_{1,0,-}\right|^{2}$ and two-photon probability $\left|C_{2,0,-}\right|^{2}$ might be reduced, simultaneously. Considering Eq. 27), we now ask a natural question: which probability is larger, because only the ratio between $\left|C_{2,0,-}\right|^{2}$ and $\left|C_{1,0,-}\right|^{4}$ makes sense.

The probability amplitudes $\left|C_{1,0,-}\right|^{2}$ and $\left|C_{2,0,-}\right|^{2}$ versus detunning $\Delta / \kappa$ are plotted in Fig. 6 for $J=0$ and $J=20 \kappa$. We analyze Fig. 6in two parameter regimes, i.e., $|\Delta| / \kappa<0.6$ and $|\Delta| / \kappa>0.6$. In the first parameter regime and around $\Delta=0$, it is clear that both $\left|C_{1,0,-}\right|^{2}$ and $\left|C_{2,0,-}\right|^{2}$ are reduced when the mode coupling is introduced. They are changed as follows

$$
\left|C_{2,0,-}\right|_{J=20 \kappa}^{2}=10^{-8}\left|C_{2,0,-}\right|_{J=0}^{2},
$$

and

$$
\left|C_{1,0,-}\right|_{J=20 \kappa}^{2}=10^{-3}\left|C_{1,0,-}\right|_{J=0}^{2} .
$$

Considering the expression of $g_{\mathrm{CCW}}^{(2)}(0)$, we find that $g_{\mathrm{CCW}}^{(2)}(0)$ is reduced by two orders of magnitude when the mode coupling is introduced, i.e., stronger photon antibunching is achieved. When $|\Delta| / \kappa>0.6$, both $\left|C_{2,0,-}\right|^{2}$ and $\left|C_{1,0,-}\right|^{4}$ are comparable for the case with mode coupling or without mode coupling, thus $g_{\mathrm{CCW}}^{(2)}(0)$ are the same for the cases with and without mode coupling in this parameter regime.

We can also obtain the value of $g_{\mathrm{CCW}}^{(2)}(0)$ via analytical solution in Eq. (A9). When $J=0$, we have

$$
\left|C_{1,0,-}\right|_{J=0}^{2}=\frac{\varepsilon^{2}\left|\Delta_{p} \Delta_{d}-g^{2}\right|^{2}}{\left|\Delta_{p}\right|^{2}\left|-2 g^{2}+\Delta_{p} \Delta_{d}\right|^{2}} .
$$

Around $\Delta=0$, the ratio of $\left|C_{1,0,-}\right|^{2}$ between $J=0$ and $J \neq 0$ is given

$$
R_{1}=\frac{\left|C_{1,0,-}\right|_{J \neq 0}^{2}}{\left|C_{1,0,-}\right|_{J=0}^{2}} \approx \frac{\kappa^{2}}{4 J^{2}},
$$
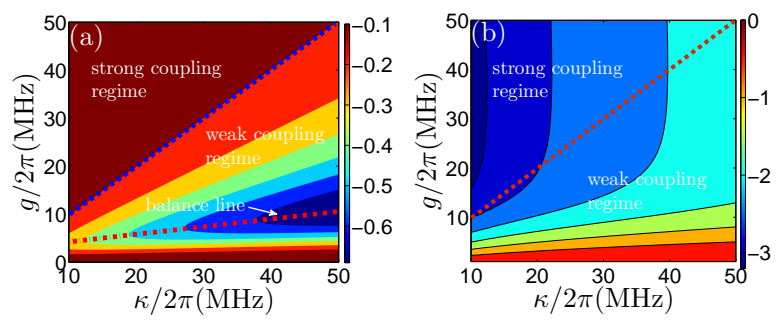

FIG. 7: (Color online) Logarithmic plot of second-order correlation functions $g_{\mathrm{CCW}}^{(2)}(0)$ of the $\mathrm{CCW}$-propagating intercavity field as functions of both cavity field decay rate $\kappa$ and DQE-field coupling strength parameter $g$ without optical mode coupling in (a) and with optical mode coupling rate $J=800 \mathrm{MHz}$ in (b). The balance line between parameter g and $\kappa$ is shown as a dash-dotted line in (a). The strong and weak DQE-field coupling regime are labeled up and below the dash line respectively. The other system parameters are: $\Delta=0, \varepsilon=\gamma_{\mathrm{a}}, \gamma_{\mathrm{a}}=1 \mathrm{MHz}$.

Similarly, we can have

$$
R_{2}=\frac{\left|C_{2,0,-}\right|_{J \neq 0}^{2}}{\left|C_{2,0,-}\right|_{J=0}^{2}} \approx \frac{\kappa^{6}}{4 J^{6}} .
$$

Then, we can obtain $g_{\mathrm{CCW}}^{(2)}(0)$ as

$$
\frac{g_{\mathrm{CCW}}^{2}(0)_{J \neq 0}}{g_{\mathrm{CCW}}^{2}(0)_{J=0}}=\frac{R_{2}}{R_{1}^{2}}=\frac{4 \kappa^{2}}{J^{2}} .
$$

When $J=20 \kappa$, we find that $g_{\mathrm{CCW}}^{(2)}(0) \approx 10^{-2}$ in the range near $\Delta=0$, which is the same as numerical calculations.

According to the above discussions, we conclude that the mode coupling results in the strong photon antibunching in the range near $\Delta=0$. To clearly show this, $g_{\mathrm{CCW}}^{(2)}(0)$ is further plotted as a function of $\kappa$ and $g$ with $J=0$ in Fig. 7 (7) and $J=800 \mathrm{MHz}$ in Fig. 7 (b). In contrast to the case of DQE-bimodal system without mode coupling [26], the strong photon antibunching appears in the larger parameter range of the DQE-field coupling strength $g$ when the mode coupling is introduced. Comparing this with no photon antibunching, i.e., $g_{\mathrm{CCW}}^{(2)}(0) \sim 1$, in the strong DQE-field coupling regime when there is no the mode-coupling, we find that a strong photon antibunching, i.e., $g_{\mathrm{CCW}}^{(2)}(0) \sim 10^{-2}$, appears in the strong DQE-field coupling regime when the mode coupling is introduced. We also find that a balance between $\kappa$ and $g$ is necessary to obtain the stronger photon antibunching in the weak DQE-field coupling regime when $J=0$. However, when the mode coupling $J$ is introduced, such balance is not required.

\section{MODE COUPLING ENHANCED ROBUSTNESS TO PURE DEPHASING}

It is well known that dephasing with the rate $\gamma_{\mathrm{p}}$ cannot be neglected in some particular DQEs, e.g., the dephasing due to electron-phonon coupling play a very important role in 

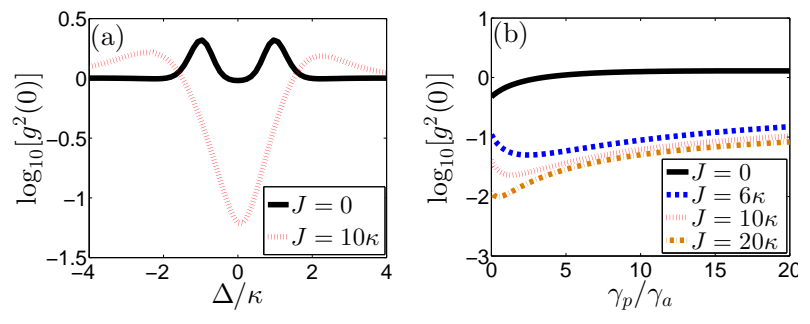

FIG. 8: (Color online) (a) The second-order correlation functions $g_{\mathrm{CCW}}^{(2)}(0)$ of the CCW-propagating intercavity fields are plotted as functions of the cavity-field detuning $\Delta / \kappa$, with $\gamma_{\mathrm{p}}=3 \gamma_{\mathrm{a}}$. The black-solid curve is for the cavity mode without coupling $(J=0)$ and red-dash-dotted curve is for strong mode-coupling $(J=10 \kappa)$. (b) The $g_{\mathrm{CCW}}^{(2)}(0)$ versus the dephasing rate $\gamma_{p} / \gamma_{a}$ with different mode-coupling strength: $J=0$ (black-solid curve), $J=6$ (bluedash curve), $J=10 \kappa$ (red-dotted curve) and $J=20 \kappa$ (yellowdotted-dash curve) at $\Delta=0$. The other system parameters for these calculations are: $\kappa=40 \gamma_{\mathrm{a}}, g=20 \gamma_{\mathrm{a}}, \varepsilon=\gamma_{\mathrm{a}}, \gamma_{\mathrm{a}}=1 \mathrm{MHz}$.

quantum dot systems. Thus, we now study the pure dephasing effect and show how it affects the statistical properties of CCW propagating optical mode in the weak DQE-field coupling regime.

We first study $g_{\mathrm{CCW}}^{(2)}(0)$ as a function of the detuning $\Delta$. Figure 3 has shown that the photon antibunching can be achieved around $\Delta=0$ for $J=0$ when there is no dephasing. However, as shown in Fig. 8 a), when a pure-dephasing, e.g., $\gamma_{\mathrm{p}}=3 \gamma_{\mathrm{a}}$, is introduced, the photon antibunching effect disappears with all the values of $g_{\text {CCIN }}^{(2)}(0)>1$ for $J=0$. Thus, if there is no the mode coupling [26], the photon antibunching is not easy to be achieved when there is the dephasing. However, when the mode coupling is introduced, e.g., $J=10 \kappa$, as shown in Fig. 8 a), a strong photon antibunching can be achieved even when there is the dephasing. The minimum value of $g_{\mathrm{CCW}}^{(2)}(0)$ corresponding to $J=10 \kappa$ is 0.06 at the point $\Delta=0$ in contrast to $g_{\mathrm{CCW}}^{(2)}(0)=1$ for $J=0$.

We further study how the dephasing affects $g_{\mathrm{CCW}}^{(2)}(0)$ in the weak DQE-field coupling regime in different mode-coupling strengths. Figure 8 b) shows that $g_{\mathrm{CCW}}^{(2)}(0)$ increases with increasing $\gamma_{\mathrm{p}}$ and quickly approaches to one for $J=0$. However, when mode coupling is introduced (as shown in Fig. 8 8 b) for three different mode-coupling strengths, e.g., $J=6 \kappa$, $J=10 \kappa$, and $J=20 \kappa$ ), the photon antibunching can still occur even with large dephasing. Interestingly, there is a minimum value of $g_{\mathrm{CCW}}^{(2)}(0)$ versus of $\gamma_{\mathrm{p}}$ for each mode-coupling strength, and this minimum value represents the optimal case for photon antibunching. We find that photon antibunching can still be achieved when $\gamma_{p}$ becomes very large. This means that the system is robust to dephasing when mode coupling is introduced. Figure 8 b) also shows that larger value of the mode-coupling strength $J$ provides a better photon antibunching when the DQE has dephasing.

In Fig. 9 a), the $g_{\mathrm{CCW}}^{(2)}(0)$ is plotted as a function of $\Delta$ and $\gamma_{\mathrm{p}}$ with $J=0$ and $J \neq 0$, e.g., $J=20 \kappa$. For $J=0$, shown
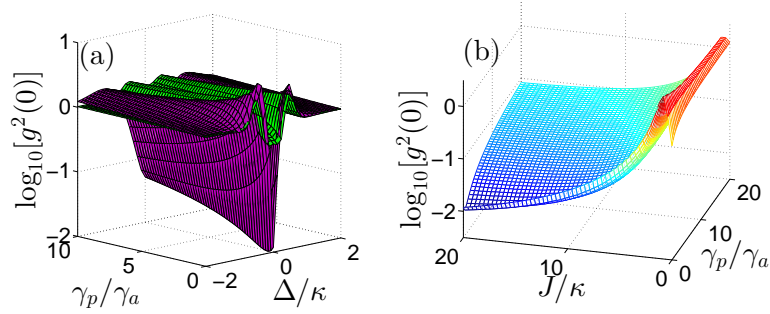

FIG. 9: (Color online) The second-order correlation functions $g_{\mathrm{CCW}}^{(2)}(0)$ of the CCW-propagating intercavity fields are plotted in (a) as functions of both the cavity field detuning $\Delta / \kappa$ and DQE dephasing rate $\gamma_{p}$, without mode coupling $J=0$ (the cyan-curved surface), and with mode coupling $J=20 \kappa$ (the magenta-curved surface). (b) shows $g_{\mathrm{CCW}}^{(2)}(0)$ versus both mode-coupling strength $J / \kappa$ and DQE dephasing rate $\gamma_{p}$ at $\Delta=0$. The other system parameters are: $\kappa=40 \gamma_{\mathrm{a}}, g=20 \gamma_{\mathrm{a}}, \varepsilon=\gamma_{\mathrm{a}}$, and $\gamma_{\mathrm{a}}=1 \mathrm{MHz}$.

as a cyan-curved surface, in Fig. 9 a), $g_{\mathrm{CCW}}^{(2)}(0)$ quickly approaches to one around $\Delta=0$ with increasing $\gamma_{p}$. This means that the antibunching is significantly affected by the dephasing. However, for $J=20 \kappa$, shown as a magenta-curved surface, in Fig. 9 a), $g_{\mathrm{CCW}}^{(2)}(0)$ is slowly increased with increasing $\gamma_{\mathrm{p}}$ around $\Delta=0$, and remains smaller than one up to $\gamma_{p}=10 \gamma_{a}$. Thus the system for the photon antibunching is very robust to the dephasing $\gamma_{\mathrm{p}}$ when the mode coupling is introduced.

In Fig. 9 b), $g_{\mathrm{CCW}}^{(2)}(0)$ is plotted as a function of $J$ and $\gamma_{\mathrm{p}}$. Figure 9 (b) shows that the larger mode-coupling strength $J$ corresponds to the smaller value of $g_{\mathrm{CCW}}^{(2)}(0)$ for a given $\gamma_{p}$, and thus a better photon antibunching. It is also obvious that all the values of $g_{\mathrm{CCW}}^{(2)}(0)$ are smaller than 0.1 for all $\gamma_{p}$ when $J / \kappa>4$. This reveals that strong photon antibunching can occur even when a large dephasing $\gamma_{p}$ is introduced (e.g., for $\gamma_{\mathrm{p}} / \gamma_{\mathrm{a}}=20$ and even larger). This is very different from the case without the mode coupling, i.e., the value of $g_{\mathrm{CCW}}^{(2)}(0)$ approaches one even with a small dephasing, as shown in Fig. 8(b) for $J=0$. Therefore, we conclude that photon antibunching is robust to the phase dephasing of the DQE when the mode coupling is introduced.

\section{ROBUSTNESS TO MODE MISMATCH AND COUPLING MISMATCH}

In previous sections, we mostly focused on the resonant interaction between the DQE and cavity modes and also the homogeneous couplings between two cavity modes and the DQE, i.e., $\omega=\omega_{a}$ and $g_{a} \equiv g_{b} \equiv g$. Let us now analyze the effect of frequency mismatch and coupling mismatch on photon antibunching.

We first study the effect of the mismatch between the frequencies $\omega$ and $\omega_{\mathrm{a}}$ of the cavity modes and the DQE on the antibunching when mode coupling is introduced. In Fig.10, for given $g_{\mathrm{a}}=g_{\mathrm{b}}=0.5 \kappa$, the second-order correlation function $g_{\mathrm{CCW}}^{(2)}(0)$ is plotted as a function of two detunings $\Delta=\omega-\omega_{d}$ 

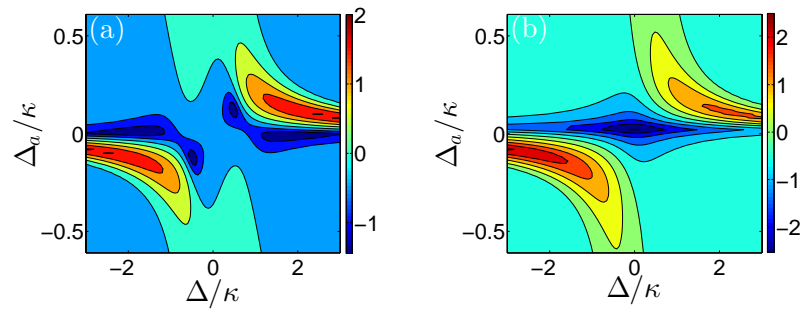

FIG. 10: (Color online) The second-order correlation functions $g_{\mathrm{CCW}}^{(2)}(0)$ of the CCW-propagating intercavity field versus both the optical mode detuning $\Delta / \kappa$ and the DQE detuning $\Delta_{a} / \kappa$, for (a) $J=0$ and (b) $J=20 \kappa$. The other system parameters are: $\kappa=40 \gamma_{\mathrm{a}}, g=20 \gamma_{\mathrm{a}}, \varepsilon=\gamma_{\mathrm{a}}$, and $\gamma_{\mathrm{a}}=1 \mathrm{MHz}$.

and $\Delta_{\mathrm{a}}=\omega_{a}-\omega_{d}$, for $J=0$ and also for $J \neq 0$, e.g., $J=20 \kappa$. We find that $g_{\mathrm{CCW}}^{(2)}(0)$ reaches its minimum value when the driving field is resonant to the DQE, e.g., $\Delta_{a}=0$. If we fix $\Delta_{a} \equiv 0$, the effect of the mismatch of the frequencies $\omega$ and $\omega_{\mathrm{a}}$ on the antibunching can be clearly observed only by changing the detuning $\Delta$. Compared with Fig. 10(a), in Fig.10(b), we find that increasing the mismatch $|\Delta|$, the value of $g_{\mathrm{CCW}}^{(2)}(0)$ increases very slowly. The value of $g_{\mathrm{CCW}}^{(2)}(0)$ increases to about 0.1 when $\Delta$ is changed from zero to a finite value for $\Delta_{\mathrm{a}}=0$. So, when mode coupling is introduced, the photon antibunching can always be obtained in any detuning $\Delta$ when the driving field is resonantly applied to the DQE, i.e., when $\Delta_{a}=0$.

We then study the effect of the mismatch of the coupling strengths $g_{a}$ and $g_{b}$ on the antibunching when the mode coupling is introduced. In Fig. 11 a) and Fig. 11 (b), for given $g_{\mathrm{a}}=0.5 \kappa$ and $\Delta=\Delta_{a}$, we plot $g_{\mathrm{CCW}}^{(2)}(0)$ as functions of $\Delta$ and $g_{\mathrm{b}}$ with $J=0$ and $J=20 \kappa$, respectively. Fig. 11(a) shows that a small range around $\Delta=0$ and $g_{\mathrm{b}}=0.4 \kappa=$ $0.8 g_{\text {a }}$ corresponds to $g_{\mathrm{CCW}}^{(2)}(0)<1$, which means photon antibunching. However, in the other range, $g_{\mathrm{CCW}}^{(2)}(0) \geq 1$, which corresponds to no photon antibunching. The minimum value appearing in Fig. 111(a) for $J=0$ is very sensitive to $g_{\mathrm{b}}$ and $\Delta$. We find that antibunching cannot be obtained even when the DQE-field coupling strength $g_{b}$ becomes large. Thus a balance between $g_{\mathrm{b}}$ and $g_{\mathrm{a}}$ is required to achieve photon antibunching when there is no mode coupling.

When the mode coupling is introduced, e.g., $J=20 \kappa$, Fig. 11 b) shows that the value of $g_{\mathrm{CCW}}^{(2)}(0)$ first decreases and then increases very slowly when increasing $g_{b}$ for $\Delta=0$. The minimum value of $g_{\mathrm{CCW}}^{(2)}(0)$ is located at $g_{\mathrm{b}}=\kappa$, which corresponds to the strongest antibunching. We find that in the weak DQE-field coupling regime, i.e., $g_{\mathrm{b}}<\kappa$, the larger value of $g_{\mathrm{b}}$ corresponds to the smaller value of $g_{\mathrm{CCW}}^{(2)}(0)$ and then better antibunching. However, in the strong-coupling regime, i.e., $g_{\mathrm{b}}>\kappa$, the value of $g_{\mathrm{CCW}}^{(2)}(0)$ increases with increasing $g_{b}$, but the value of $g_{\mathrm{CCW}}^{(2)}(0)$ is still very small, i.e., $g_{\mathrm{CCW}}^{(2)}(0) \leq 0.0005$ in the given parameter regime.

To further find the effect of the mismatch between $g_{\mathrm{a}}$ and $g_{\mathrm{b}}$
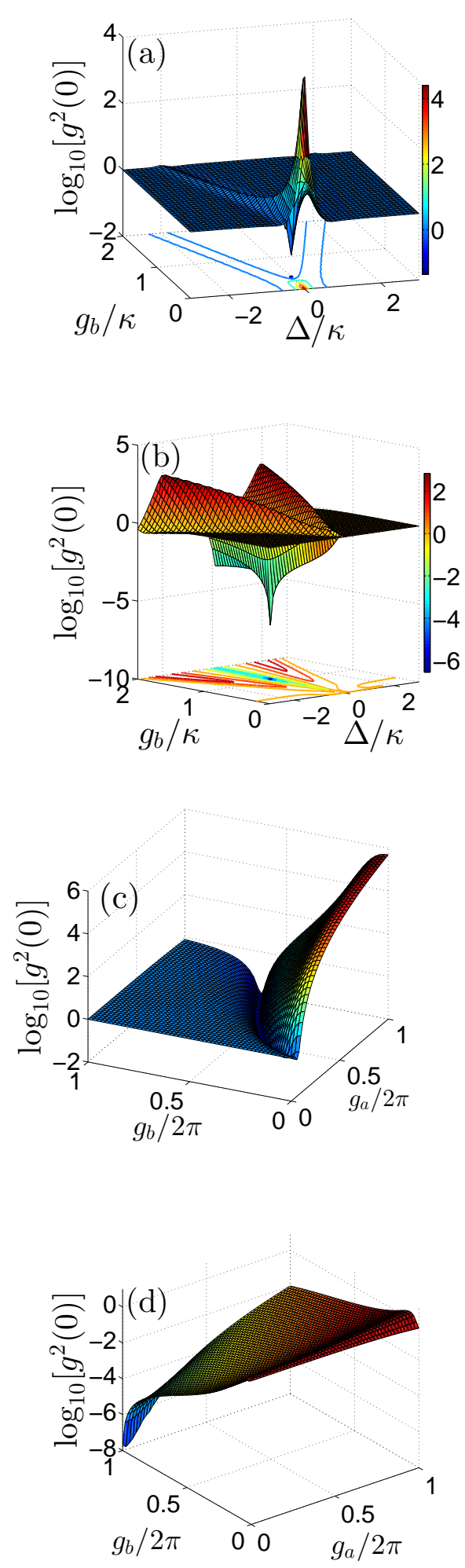

FIG. 11: (Color online) The second-order correlation functions $g_{\mathrm{CCW}}^{(2)}(0)$ of the CCW-propagating intercavity field are plotted as functions of both cavity field detuning $\Delta / \kappa$ and DQE-field coupling rate $g_{b} / \kappa$ (without mode coupling $J=0$ and with mode coupling $J=20 \kappa$ ) for $g=0.5 \kappa$, as shown in (a) and (b), respectively. $g_{\mathrm{CCW}}^{(2)}(0)$ is plotted as functions of coupling strength $g_{\mathrm{a}}$ and $g_{\mathrm{b}}$ (without mode coupling $J=0$ and with mode coupling $J=20 \kappa$ ) for $\Delta=0$, as shown in (c) and (d), respectively. The other system parameters are: $\kappa=40 \gamma_{\mathrm{a}}, \varepsilon=\gamma_{\mathrm{a}}$, and $\gamma_{\mathrm{a}}=1 \mathrm{MHz}$. 
on the photon antibunching, $g_{\mathrm{CCW}}^{(2)}(0)$ is plotted in Fig.11(c) and in Fig. 11 d) as a function of $g_{\mathrm{a}}$ and $g_{\mathrm{b}}$ for $\Delta=\Delta_{a}=0$, with $J=0$ and $J \neq 0$, e.g., $J=20 \kappa$. For $J=0$, Fig. 11 (c) clearly shows the antibunching can only occur in a narrow range which needs a balance between $g_{\mathrm{a}}$ and $g_{\mathrm{b}}$ as previous discussions. In most of the parameter range of $g_{a}$ and $g_{b}$, the value of $g_{\mathrm{CCW}}^{(2)}(0)$ satisfies the condition $g_{\mathrm{CCW}}^{(2)}(0) \geq 1$, which means that there is no photon antibunching. However, when mode coupling is introduced, e.g., $J=20 \kappa$, the balance between $g_{\mathrm{a}}$ and $g_{\mathrm{b}}$ is not required. Figure 11d) also shows that the value of $g_{\mathrm{CCW}}^{(2)}(0)$ increases when increasing both $g_{\mathrm{a}}$ and $g_{b}$ for given other parameters. From Fig.11d), we also find a very small value $10^{-8}$ of $g_{\mathrm{CCW}}^{(2)}(0)$ can be achieved at $g_{b}=\kappa$ when $g_{\mathrm{a}}=0$, i.e., the CCW mode is decoupled from the DQE.

Therefore, we conclude that the strong robustness gainst mismatches discussed above can be achieved when mode coupling is introduced. A balance, between $g_{a}$ and $g_{b}$ for achieving better photon antibunching for the case without mode coupling is not required when mode coupling is introduced. In particular, we find that the mismatch between $g_{a}$ and $g_{b}$ can play a positive role to produce photon antibunching when mode coupling is introduced.

\section{CONCLUSIONS}

In summary, we have studied photon antibunching in a system consisting of a bimodal WGMC and a DQE. Two modes in the WGMC are coupled via a scatterer. We mainly study the effect of the mode coupling on the photon antibunching. it is known that photon antibunching cannot occur in a system where a single-mode cavity field is weakly coupled to a DQE. However, we find that a very strong antibunching can be achieved in the weak DQE-field coupling regime, when mode coupling is introduced.

Comparing with the case when the DQE is coupled to two modes of the bimodal cavity without mode coupling [26], when mode coupling is introduced, we find that: (i) a strong photon antibunching can be achieved in a larger parameter regime including both the weak and strong DQE-field coupling regime; (ii) the minimum value of second-order correlation function can be reduced up to several orders of magnitude even in the weak DQE-field coupling regime; that is, a strong photon antibunching can be achieved; (iii) the system is more robust to phase dephasing of the DQE; (iv) the system is also more robust to the frequency mismatch between the cavity modes and the DQE, as well as the mismatch of the coupling strengths between two modes of the cavity and the DQE.

Our study shows that the system of two coupled cavity fields interacting with a DQE could be a good quantum device for producing antibunched photons. Our studies support a platform to achieve single-photon sources on chip with robustness against fabrication imperfections and several mismatches in the system.

\section{ACKNOWLEDGEMENT}

Y.X.L. is supported by the National Basic Research Program of China 973 Program under Grant No. 2014CB921401, the National Natural Science Foundation of China under Grant Nos. 61328502, 61025022, the Tsinghua University Initiative Scientific Research Program, and the Tsinghua National Laboratory for Information Science and Technology (TNList) Cross-discipline Foundation. FN is partly supported by the RIKEN iTHES Project, the MURI Center for Dynamic Magneto-Optics, a Grant-in-Aid for Scientific Research (S), and the ImPACT Program of JST.

\section{Appendix A: Analytical solutions of the second-order correlation function with weak pump limit}

In the limit of a weak-driving field, the total excitation number of the system is assumed no more than two. Using the ansatz given in Eqs. (22) and (23) and combining with Eqs. (24)-(26), the coefficients $C_{\mathrm{i}, \mathrm{j}, \pm}$ in the steady state can be obtained via the Schrödinger equation by set $\partial|\varphi\rangle / \partial t=0$. With the zero temperature approximation and neglecting the pure dephasing of the DOE, we finally obtain the following set of linear equations

$$
\begin{aligned}
0= & \left(\Delta-i \frac{\kappa}{2}\right) C_{1,0,-}+J C_{0,1,-}+g C_{0,0,+}+\varepsilon, \quad \text { (A1) } \\
0= & \left(\Delta-i \frac{\kappa}{2}\right) C_{0,1,-}+J C_{1,0,-}+g C_{0,0,+}, \quad \text { (A2) } \\
0= & \left(\Delta-i \frac{\gamma_{a}}{2}\right) C_{0,0,+}+g C_{1,0,-}+g C_{0,1,-}, \quad \text { (A3) } \\
0= & (2 \Delta-i \kappa) C_{2,0,-}+\sqrt{2} J C_{1,1,-} \\
& +\sqrt{2} g C_{1,0,+}+\sqrt{2} \varepsilon C_{1,0,-}, \\
0= & (2 \Delta-i \kappa) C_{0,2,-}+\sqrt{2} J C_{1,1,-}+\sqrt{2} g C_{0,1,+} \text { (A5) } \\
0= & (2 \Delta-i \kappa) C_{1,1,-}+\sqrt{2} J C_{0,2,-}+\sqrt{2} J C_{2,0,-} \\
& +g C_{0,1,+}+g C_{1,0,+}+\varepsilon C_{0,1,-}, \\
0 & {\left[2 \Delta-i\left(\frac{\kappa+\gamma_{a}}{2}\right)\right] C_{1,0,+}+J C_{0,1,+} } \\
& +\sqrt{2} g C_{2,0,-}+g C_{1,1,-}+\varepsilon C_{0,0,+,} \\
0 & {\left[2 \Delta-i\left(\frac{\kappa+\gamma_{a}}{2}\right)\right] C_{0,1,+}+J C_{1,0,+} } \\
& +g C_{1,1,-}+\sqrt{2} g C_{0,2,-} .
\end{aligned}
$$

Due to the weak pump limit $\varepsilon \rightarrow 0$, we can assume $C_{0,0,-} \rightarrow$ 1 , and one additional equation, namely $\varepsilon C_{1,0,-}=0$, is irrelevant to the problem. The Eqs. A1 -A8 are now closed (i.e., eight equations for eight parameters). Thus, it is possible to obtain the analytical solutions for all the coefficients $C_{i, j, \pm}$. However, the solution is cumbersome, so only $C_{1,0,-}$ and $C_{2,0,-}$ are given 


$$
\begin{aligned}
C_{1,0,-} & =\frac{\varepsilon\left(\Delta_{\mathrm{p}} \Delta_{\mathrm{d}}-g^{2}\right)}{\left(J-\Delta_{\mathrm{p}}\right) C_{1}}, \\
C_{2,0,-} & =\frac{\sqrt{2} \varepsilon^{2} L}{C_{3}}
\end{aligned}
$$

where

$$
\begin{aligned}
C_{1} & =-2 g^{2}+J \Delta_{\mathrm{d}}+\Delta_{\mathrm{p}} \Delta_{\mathrm{d}}, \\
C_{2} & =\left(J+\Delta_{\mathrm{p}}\right)^{2}+\Delta_{\mathrm{d}}\left(J+\Delta_{\mathrm{p}}\right)-2 g^{2}, \\
C_{3} & =2\left(J-\Delta_{\mathrm{p}}\right)^{2} C_{1} C_{2},
\end{aligned}
$$

$$
\begin{aligned}
L= & \Delta_{\mathrm{p}}^{3} \Delta_{\mathrm{d}}+\Delta_{\mathrm{p}}^{2} \Delta_{\mathrm{d}}^{2}+J \Delta_{\mathrm{p}}^{2} \Delta_{\mathrm{d}} \\
& -2 \Delta_{\mathrm{p}} \Delta_{\mathrm{d}} g^{2}-2 J \Delta_{\mathrm{p}} g^{2}+g^{4}, \\
\Delta_{\mathrm{p}}= & \Delta-i \frac{\kappa}{2} \\
\Delta_{\mathrm{d}}= & \Delta-i \frac{\gamma_{\mathrm{a}}}{2} .
\end{aligned}
$$

We substitute Eqs. A9 and (A10) into Eq. (24), and then can derive the analytical solutions of the $g_{\mathrm{CCW}}^{(2)}(0)$ in the form of Eq. (27).
[1] A. J. Shields, Nat. Photonics 1, 215 (2007).

[2] S. Buckley, K. Rivoire, and J. Vučković, Rep. Prog. Phys. 75, 126503 (2012).

[3] A. Scherer, B. C. Sanders, and W. Tittel, Opt. Express 19, 3004 (2011).

[4] N. Lütkenhaus, Norbert, Phys. Rev. A 61, 52304 (2000).

[5] D. M. Greenberger, M. A. Horne, and A. Zeilinger, Phys. Today 46, 22 (1993).

[6] V. Giovannetti, S. Lloyd, and L. Maccone, Science 306, 1330 (2004).

[7] G. S. Agarwal, R. W. Boyd, E. M. Nagasako, and S. J. Bentley, Phys. Rev. Lett. 86, 1389 (2001).

[8] D. Fattal, K. Inoue, J. Vučković, C. Santori, G. Solomon, and Y. Yamamoto, Phys. Rev. Lett. 92, 037903 (2004).

[9] V. Giovannetti, S. Lloyd, and L. Maccone, Nature 412, 417 (2001).

[10] A. I. Lvovsky, B. C. Sanders, and W. Tittel, Nat. Photonics 3, 706 (2009).

[11] A. E. Kozhekin, K. Molmer, and E. Polzik, Phys. Rev. A 62, 33809 (2000).

[12] J. I. Cirac, P. Zoller, H. J. Kimble, and H. Mabuchi, Phys. Rev. Lett. 78, 16 (1997).

[13] X. Maitre, E. Hagley, G. Nogues, C. Wunderlich, P. Goy, M. Brune, J. M. Raimond, and S. Haroche, Phys. Rev. Lett. 79, 769 (1997).

[14] M. A. Nielsen and I. L. Chuang, Quantum Computation and Quantum Information. (Cambridge: Cambridge University Press, 2000)

[15] T. Jennewein, M. Barbieri, and A. G. White, J. Mod. Opt. 58, 276 (2011).

[16] I. Bulutas and F. Nori, Science 326, 108 (2009).

[17] I. M. Georgescu, S. Ashhab, and F. Nori, Rev. Mod. Phys. textbf86, 153 (2014).

[18] T. A. Fulton and G. J. Dolan, Phys. Rev. Lett. 59, 109 (1987).

[19] M. A. Kastner, Rev. Mod. Phys. 64, 849 (1992).

[20] K. K. Likharev, Proc. IEEE 87, 606 (1999).

[21] A. Imamoḡlu, H. Schmidt, G. Woods, and M. Deutsch, Phys. Rev. Lett. 79, 1467 (1997).

[22] P. Grangier, D. F. Walls, and K. M. Gheri, Phys. Rev. Lett. 81, 2833 (1998).

[23] T. Peyronel, O. Firstenberg, Q.-Y. Liang, S. Hofferberth, A. V Gorshkov, T. Pohl, M. D. Lukin, and V. Vuletić, Nature 488, 57 (2012).

[24] S. Ferretti, V. Savona, and D. Gerace, New J. Phys. 15, 025012 (2013).

[25] K. M. Birnbaum, A. Boca, R. Miller, A. D. Boozer, T. E.
Northup, and H. J. Kimble, Nature 436, 87 (2005).

[26] B. Dayan, A. S. Parkins, T. Aoki, E. P. Ostby, K. J. Vahala, and H. J. Kimble, Science 319, 1062 (2008).

[27] A. Faraon, I. Fushman, D. Englund, N. Stoltz, P. Petroff, and J. Vučković, Nat. Phys. 4, 859 (2008).

[28] C. Lang, D. Bozyigit, C. Eichler, L. Steffen, J. M. Fink, A. A. Abdumalikov, M. Baur, S. Filipp, M. P. da Silva, A. Blais, and A. Wallraff, Phys. Rev. Lett. 106, 243601 (2011).

[29] A. J. Hoffman, S. J. Srinivasan, S. Schmidt, L. Spietz, J. Aumentado, H. E. Türeci, and A. A. Houck, Phys. Rev. Lett. 107, 053602 (2011).

[30] I. Buluta, S. Ashhab, and F. Nori, Rep. Prog. Phys. 74, 104401 (2011).

[31] J. Q. You and F. Nori, Nature 474, 589 (2011).

[32] Z.-L. Xiang, S. Ashhab, J. Q. You and F. Nori, Rev. Mod. Phys. 85, 623 (2013).

[33] A. Ridolfo, M. Leib, S. Savasta, and M. J. Hartmann, Phys. Rev. Lett. 109, 193602 (2012).

[34] Y. X. Liu, X. W. Xu, A. Miranowicz, and F. Nori, Phys. Rev. A 89, 043818 (2014).

[35] X. W. Xu, Y. J. Li, and Y. X. Liu, Phys. Rev. A 87, 025803 (2013).

[36] J. Q. Liao, H. K. Cheung, and C. K. Law, Phys. Rev. A 85, 025803 (2012).

[37] J. Q. Liao and C. K. Law, Phys. Rev. A 87, 043809 (2013).

[38] X. Y. Lü, W. M. Zhang, S. Ashhab, Y. Wu, and F. Nori, Sientific Rep. 3, 2943 (2013).

[39] T. C. H. Liew and V. Savona, Phys. Rev. Lett. 104, 183601 (2010).

[40] M. Bamba, A. Imamoğlu, I. Carusotto, and C. Ciuti, Phys. Rev. A 83, 021802 (2011)

[41] X.-W. Xu and Y.-J. Li, J. Phys. B 46, 035502 (2013).

[42] K. Stannigel, P. Komar, S. J. M. Habraken, S. D. Bennett, M. D. Lukin, P. Zoller, and P. Rabl, Phys. Rev. Lett. 109, 013603 (2012).

[43] M. Ludwig, A. H. Safavi-Naeini, O. Painter, and F. Marquardt, Phys. Rev. Lett. 109, 063601 (2012).

[44] O. Kyriienko, I. A. Shelykh, and T. C. H. Liew, arXiv: 1403.7441 v1 (2014).

[45] Y. L. Liu, Z. P. Liu, J. Zhang, and Y. X. Liu, arXiv:1407.3036v1 (2014).

[46] D. Gerace and V. Savona, Phys. Rev. A 89, 031803 (2014).

[47] H. Flayac and V. Savona, Phys. Rev. A 88, 033836 (2013).

[48] J. Zhu, Ş. K. Özdemir, Y. Xiao, L. Li, L. He, D. Chen, and L. Yang, Nat. Photonics 4, 46 (2009).

[49] L. He, Ş. K. Özdemir, J. Zhu, W. Kim, and L. Yang, Nat. Nan- 
otechnol. 6, 428 (2011).

[50] F. Vollmer and L. Yang, Nanophotonics 1, 267 (2012).

[51] J. Zhu, Ş. K. Özdemir, L. He, and L. Yang, Opt. Express 18, 23535 (2010).

[52] J. Zhu, Ş. K. Özdemir, L. He, D.-R. Chen, and L. Yang, Opt. Express 19, 16195 (2011).

[53] L. He, Ş. K. Ödemir, J. Zhu, and L. Yang, Phys. Rev. A 82, 053810 (2010).

[54] L. He, Ş. K. Ödemir, J. Zhu, F. Monifi, H. Yilmaz, and L. Yang, New J. Phys. 15, 073030 (2013).

[55] K. Srinivasan and O. Painter, Nature 450, 862 (2007).

[56] K. Srinivasan and O. Painter, Phys. Rev. A 75, 023814 (2007).

[57] A. Majumdar, M. Bajcsy, A. Rundquist, and J. Vučković, Phys. Rev. Lett. 108, 183601 (2012).

[58] W. Zhang, Z. Yu, Y. Liu, and Y. Peng, Phys. Rev. A 89, 043832
(2014).

[59] H. J. Carmichael, An Open Systems Approach to Quantum Optics (Springer-Verlag, Berlin, 1993).

[60] H. J. Carmichael, Statistical Methods in Quantum Optics I:Master Equations and Fokker-Planck Equations (SpringerVerlag, Berlin, 2003).

[61] J. Hauss, A. Fedorov, S. André V. Brosco, C. Hutter, R. Kothari, S. Yeshwanth, A. Shnirman, and G. Schön, New J. Phys. 10, 095018 (2008).

[62] Ş. K. Ödemir, J. Zhu, X. Yang, B. Peng, H. Yilmaz, L. He, F. Monifi, G. L. Long and L. Yang. arXiv:1401.2033.

[63] M. J. Collett and C.W. Gardiner, Phys. Rev. A 30, 1386 (1984).

[64] C.W. Gardiner and M. J. Collett, Phys. Rev. A 31, 3761 (1985). 